\title{
Designing Interactive Secure Systems: Workshop at British $\mathrm{HCl} 2012$
}

\author{
Shamal Faily \\ Department of Computer Science \\ University of Oxford \\ shamal.faily@cs.ox.ac.uk
}

\author{
Ivan Fléchais \\ Department of Computer Science \\ University of Oxford \\ ivan.flechais@cs.ox.ac.uk
}

\author{
Lizzie Coles-Kemp \\ Information Security Group \\ Royal Holloway University of London \\ lizzie.coles-kemp@rhul.ac.uk
}

\begin{abstract}
Preface to the proceedings of Designing Interactive Secure Systems: Workshop at British $\mathrm{HCl} 2012$, held at the University of Birmingham on 11th September 2012
\end{abstract}

British HCI,HCI 2012,Designing Interactive Secure Systems, DISS2012

\section{INTRODUCTION}

Welcome to the proceedings of the inaugural workshop on Designing Interactive Secure Systems (DISS 2012). This workshop was held in conjunction with the 26th BCS Interaction Specialist Group Conference on People and Computers at the University of Birmingham on September 11th 2012.

In recent years, the field of usable security has attracted researchers from $\mathrm{HCl}$ and Information Security, and led to a better understanding of the interplay between human factors and security mechanisms. Despite these advances, designing systems which are both secure in, and appropriate for, their contexts of use continues to frustrate both researchers and practitioners. One reason is a misunderstanding of the role that $\mathrm{HCl}$ can play in the design of secure systems. A number of eminent security researchers and practitioners continue to espouse the need to treat people as the weakest link, and encourage designers to build systems that Homer Simpson can use. Unfortunately, treating users as a problem can limit the opportunities for innovation when people are engaged as part of a solution. Similarly, while extreme characters (such as Homer) can be useful for envisaging different modes of interaction, when taken out of context they risk disenfranchising the very people the design is meant to support.

Better understanding the relationship between human factors and the design of secure systems is an important step forward, but many design research challenges still remain. There is growing evidence that $\mathrm{HCl}$ design artefacts can be effective at supporting secure system design, and that some alignment exists between $\mathrm{HCl}$, security, and software engineering activities. However, more is needed to understand how broader insights from the interactive system design and user experience communities might also find traction in secure design practice. For these insights to lead to design practice innovation, we also need usability and security evaluation activities that better support interaction design, together with software tools that augment, rather than hinder, these design processes. Last, but not least, we need to share experiences and anecdotes about designing usable and secure systems, and reflect on the different ways of performing and evaluating secure interaction design research.

The objective of this workshop was to act as a forum for those interested in the design of interactive secure systems. By bringing together a like-minded community of researchers and practitioners, we aimed to share knowledge gleaned from recent research, as well as experiences designing secure and usable systems in practice. In doing so, this workshop became a crucible for building an interactive secure system design community, and forming collaborative partnerships to progress many of the aforementioned challenges.

Although this was the inaugural edition of this particular workshop, it built on the success of recent workshops in usable security at BCS HCl 2010 and $\mathrm{BCS} \mathrm{HCl}$ 2011. This workshop also drew on the need highlighted at last year's NIST sponsored event on Security and Usable Security Aligned for Good Engineering for building bridges between different design disciplines, and providing a forum for sharing 
anecdotal experiences about the design of usable and secure systems.

The aim of British $\mathrm{HCl} 2012$ was to return to the conference's founding theme of People and Computers. Like the $\mathrm{HCl}$ field in general, the growing diversity of work in $\mathrm{HCl}$-Security and Interactive System Design made the running of this workshop a timely opportunity to return to the design problems that motivated early work in these areas.

\section{TECHNICAL PROGRAMME}

In our call for papers, we invited 4-page position papers that would provoke discussion about the design of interactive secure systems. Our suggested list of topics included design techniques for socio-technical systems, technology for supporting interactive secure system design, usable and secure system evaluation, and experience reports.

The review process for each submission was rigorous. To select the technical programme, we were fortunate to be able to draw upon a panel of international experts; their expertise spanned the spectrum of interactive secure system design, from arts/design through to security engineering. Each paper submission received at least three reviews, although the vast majority received four. In addition to assessing the quality of work, reviewers were also asked to highlight areas they felt would provoke interesting discussion during the workshop itself. As such, these proceedings not only represent the efforts of the paper authors, but also the reviewers who provided detailed reviews and insightful suggestions to make sure authors get the most out of the DISS workshop experience.

\section{ACKNOWLEDGEMENTS}

This workshop would not have been possible without the hard work and dedication of our programme committee and external reviewers who, despite managing a slew of other commitments, still managed to find time to provide timely, high quality reviews to the paper authors. We are also grateful to the paper authors for contributing their work and participating in the workshop programme itself. Finally, we would like to thank the organisers of British $\mathrm{HCl} 2012$ for hosting us, and the EU FP 7 webinos project for their sponsorship of this event.

\section{ORGANISING AND PROGRAMME COMMITTEE}

\subsection{Organising Committee}

\author{
Shamal Faily \\ Ivan Fléchais \\ Lizzie Coles-Kemp
}

\subsection{Programme Committee}

\author{
Yoko Akama \\ Henrik Artman \\ Debi Ashenden \\ Steffen Bartsch \\ Joel Brynielsson \\ Lynne Coventry \\ Paul Dunphy \\ Richard Ford \\ Peter Hall \\ Tristan Henderson \\ Christina Hochleitner \\ Marina Jirotka \\ Mike Just \\ Ronald Kainda \\ Linda Little \\ John Lyle \\ Vicki Moulder \\ Simon Parkin \\ Karen Renaud \\ Mary Theofanos \\ Joss Wright
}

\subsection{External Reviewers}

Joe Loughry

Fernando Muradas 